\title{
openheart Residual cardiovascular risk in individuals on lipid-lowering treatment: quantifying absolute and relative risk in the community
}

\author{
Wolfgang Lieb, ${ }^{1,2}$ Danielle M Enserro, ${ }^{1,3,4}$ Martin G Larson, ${ }^{1,4}$ \\ Ramachandran S Vasan ${ }^{1,3,5}$
}

\begin{abstract}
- Additional material is published online only. To view please visit the journal online (http://dx.doi.org/10.1136/ openhrt-2017-000722).
\end{abstract}

To cite: Lieb W, Enserro DM, Larson MG, et al. Residual cardiovascular risk in individuals on lipid-lowering treatment: quantifying absolute and relative risk in the community. Open Heart 2018;5:e000722. doi:10.1136/ openhrt-2017-000722

Received 11 September 2017 Revised 14 December 2017 Accepted 29 December 2017

\section{Check for updates}

${ }^{1}$ Framingham Heart Study, Framingham, Massachusetts, USA

${ }^{2}$ Institute of Epidemiology, Christian Albrechts University Kiel, Kiel, Germany ${ }^{3}$ Section of Preventive Medicine and Epidemiology, Boston University School of Medicine, Boston, Massachusetts, USA ${ }^{4}$ Department of Biostatistics, Boston University School of Public Health, Boston, Massachusetts, USA ${ }^{5}$ Department of Epidemiology, Boston University School of Public Health, Boston, Massachusetts, USA

Correspondence to Dr Ramachandran S Vasan; vasan@bu.edu and Dr Wolfgang Lieb; Wolfgang.lieb@epi.unikiel.de

\section{ABSTRACT}

Objective The residual cardiovascular disease (CVD) risk in individuals on long-term lipid-lowering treatment (LLT) in the general population is not well described.

Methods We estimated absolute CVD risks by age and sex for different categories of low-density lipoprotein cholesterol (LDL-C) levels, stratified by LLT status, and assessed subclinical carotid atherosclerosis in 3012 Framingham Study participants (mean age, 58.4 years; $55 \%$ women) free of CVD. Individuals were categorised into five groups: (1) LDL-C $<100 \mathrm{mg} / \mathrm{dL}$ without LLT; (2) $\mathrm{LDL}-\mathrm{C} \geq 100 \mathrm{mg} / \mathrm{dL}$ to $<130 \mathrm{mg} / \mathrm{dL}$ without LLT; (3) LDL-C $<130 \mathrm{mg} / \mathrm{dL}$ on LLT; (4) LDL-C $\geq 130 \mathrm{mg} / \mathrm{dL}$ without LLT; and (5) $\mathrm{LDL}-\mathrm{C} \geq 130 \mathrm{mg} / \mathrm{dL}$ on LLT.

Results Individuals in groups 3-5 had significantly more carotid atherosclerosis compared with group 1 . During follow-up (median, 13.7 years), 548 CVD events occurred. Individuals on LLT (groups 3 and 5) had substantial residual CVD risk (26.7 (95\% Cl 19.5 to 34.0$)$ and $24.1(95 \% \mathrm{Cl} 16.2$ to 31.9$)$ per 1000 person-years, respectively), representing approximately three times the risk for untreated individuals with $\mathrm{LDL}<100 \mathrm{mg} / \mathrm{dL}$ (group 1: 9.0 (95\% Cl 6.8 to 11.3) per 1000 person-years). Absolute CVD risks rose with age and were slightly greater in men than in women. After adjustment for traditional risk factors, groups 3-5 displayed increased hazards for CVD $(\mathrm{HR}=1.47,1.42$ and 1.54, respectively) compared with group 1. Further adjustment for carotid atherosclerosis modestly attenuated these results.

Conclusions There is substantial residual CVD risk in individuals on LLT, compared with participants with optimal LDL-C $(<100 \mathrm{mg} / \mathrm{dL})$, even when LDL-C levels $<130 \mathrm{mg}$ / $\mathrm{dL}$ are reached.

\section{INTRODUCTION}

Based on mounting evidence from randomised controlled trials in both primary and secondary prevention settings, ${ }^{1-4}$ lipid-lowering treatment (LLT; pharmacological and lifestyle) is a fundamental pillar of preventive cardiology.

Relatively little evidence exists regarding the remaining absolute disease risk in individuals on LLT, and there is currently an active debate regarding the broad application of

\section{Key questions}

What is already known about this subject?

- Lipid-lowering treatment (LLT) reduces cardiovascular disease (CVD) risk and is, therefore, a fundamental pillar of preventive cardiology.

- The residual CVD risk in individuals on long-term LLT in the general population is not well described.

What does this study add?

- In a large community-based sample, we quantified subclinical disease burden at the carotid arteries and provide absolute CVD risk estimates for different categories of low-density lipoprotein cholesterol (LDL-C) levels, representing different treatment intensities of LLT.

- Individuals on LLT had greater subclinical disease burden and displayed a substantial residual CVD risk, approximately tripling the risk of untreated individuals with $\mathrm{LDL}-\mathrm{C}<100 \mathrm{mg} / \mathrm{dL}$.

- This residual risk in individuals on LLT in the general population is partly explained by an adverse profile of other CVD risk factors and in part by carotid subclinical atherosclerosis in these people.

How might this impact on clinical practice?

- Our observations emphasise the necessity to closely monitor all standard CVD risk factors in individuals on LLT, even when LDL levels are lowered effectively.

statins. ${ }^{5-7}$ While most people would agree that there is a residual risk, the magnitude of this risk and its variation with age, sex and lipid level categories on LLT (representing different intensities of LLT) is not well quantified, particularly not in the community. This is because most randomised controlled trials have strict inclusion and exclusion criteria and might, therefore, not ideally mirror the situation in the community. Furthermore, the extent to what the residual cardiovascular risk is attributable to subclinical vascular disease 
or to alterations in the neurohormonal or inflammatory milieu is also not well described. A detailed quantification of the residual cardiovascular disease (CVD) risk in the community and a better understanding of its biology would be essential to lower the burden of CVD. Part of the mechanisms by which dyslipidaemia promotes CVD risk is by enhancing the substrate of subclinical atherosclerosis and by altering the neurohormonal and inflammatory milieu.

We hypothesised that (1) although LLT lowers CVD risk, individuals on LLT experience substantial residual CVD risk that exceeds the risk observed in untreated individuals with similar levels of low-density lipoprotein cholesterol (LDL-C), and (2) this residual CVD risk is in part due to the burden of subclinical atherosclerosis and due to systemic inflammation and neurohormonal activation.

Accordingly, we assessed (1) the burden of subclinical disease at the carotid arteries cross-sectionally and (2) the risk for incident CVD prospectively, associated with different levels of LDL-C in a large community-based sample, stratified by the use of LLT.

\section{METHODS}

\section{Study sample}

The present analyses were based on data from attendees at the sixth examination cycle of the Framingham Offspring cohort (1995-1998). ${ }^{8}$ A total of 520 individuals were excluded for reasons detailed in the online supplementary material, resulting in a sample size of $n=3012$. For 95 individuals, information regarding the subclinical disease burden of the carotid artery (detailed below) was missing, resulting in a sample size of $n=2917$ for the analyses related to subclinical carotid disease burden. Circulating $\mathrm{C}$ reactive protein (CRP) concentrations and plasma levels of B-type natriuretic peptide (BNP) were available in 2811 participants. All participants provided written informed consent.

\section{Laboratory measurements}

Lipid traits, including blood levels of total cholesterol, high-density lipoprotein (HDL) cholesterol and triglycerides, were measured in fasting participants using standardised assays. LDL-C was calculated using the Friedewald formula in individuals with triglyceride levels $<400 \mathrm{mg}$ / dL. ${ }^{9}$ Measurements of CRP and BNP are described in the online supplementary material.

\section{Ultrasound of the carotid artery}

Ultrasonographic measurements of the carotid arteries on both sides were performed using a $7.5 \mathrm{MHz}$ transducer and a $5.0 \mathrm{MHz}$ transducer for images of the common and of the internal carotid arteries, respectively, on a Toshiba SSH-140 A machine, following a standardised protocol. ${ }^{10-12}$ More details regarding the ultrasound measurements are provided in the online supplementary material.
'Carotid ultrasound abnormality' was defined as (1) increased $\left(\geq 80^{\text {th }}\right.$ sex-specific percentile $)$ intima media thickness (IMT), a combined standardised measure including information from the internal and common carotid arteries; (2) an extreme increase of the common carotid IMT $\geq 1 \mathrm{~mm}$; or (3) significant stenosis ( $\geq 25 \%$ narrowing) of the common or internal carotid arteries, consistent with prior publications. ${ }^{13}{ }^{14}$ We chose this combined parameter in order to reflect both alterations in IMT (a measure of overall atherosclerotic plaque burden) and the presence of arterial stenoses (a measure of obstructive atherosclerosis).

\section{Adjudication of incident CVD events}

Framingham participants are under regular surveillance for new-onset CVD events. More details regarding the adjudication of events are provided in the online supplementary material.

\section{Statistical analyses}

Based on the intake of lipid-lowering medications and the blood LDL levels determined at the sixth examination cycle, participants were assigned to one of five mutually exclusive groups.

1. usual LDL-C $<100 \mathrm{mg} / \mathrm{dL}$, not on LLT

2. usual LDL-C $\geq 100 \mathrm{mg} / \mathrm{dL}$ to $<130 \mathrm{mg} / \mathrm{dL}$, not on LLT

3. treated LDL-C $<130 \mathrm{mg} / \mathrm{dL}$ on LLT

4. usual LDL-C $\geq 130 \mathrm{mg} / \mathrm{dL}$, not on LLT

5. treated LDL-C $\geq 130 \mathrm{mg} / \mathrm{dL}$ on LLT.

There were very few individuals $(\mathrm{n}=58)$ with LDL-C $<100 \mathrm{mg} / \mathrm{dL}$ on LLT; therefore no separate category was created for these individuals. We chose these cut points taking into account different target lipid levels and the presence versus absence of LLT, thereby ensuring that the strata yielded an adequate number of events to enable analyses with reasonable statistical power.

To quantify the baseline risks in the different LDL groups, we calculated the 10-year probability of a CVD event ${ }^{15}$ for each participant and displayed these event probabilities as boxplots according to LDL group. Treated individuals (groups 3 and 5) were compared versus untreated individuals (groups 1, 2 and 4) with respect to their 10-year CVD event probabilities using analysis of variance.

\section{Subclinical disease burden at the carotid arteries by LDL group}

First, we calculated the ORs for the binary trait 'carotid ultrasound abnormality' (defined as detailed above, including information on IMT and on stenoses) for each LDL group, using group 1 as the referent, in age-and sex-adjusted as well as multivariable-adjusted logistic regression models. The multivariable models included age, sex, systolic blood pressure, antihypertensive medication, smoking and diabetes mellitus. Second, we compared the odds for 'carotid ultrasound abnormality' in individuals on LLT (group 5 and group 3) with the odds for 'carotid ultrasound abnormality' in untreated 
individuals with similar LDL levels (group 4 and groups $1+2$ combined, respectively) using age- and sex-adjusted as well as multivariable-adjusted logistic regression models. We focused on 'carotid ultrasound abnormality' as the primary subclinical disease trait because subclinical alterations of the carotid artery are reported to be strongly associated with increased LDL levels ${ }^{16}$ and the selected trait 'carotid ultrasound abnormality' includes both information from IMT and of relevant stenoses of the carotid arteries. ${ }^{13} 14$

\section{Association of LDL category with incident CVD}

We performed several analyses to describe the residual cardiovascular risk associated with different levels of LDL-C, stratified by LLT status, age group and sex. First, the unadjusted survival free of CVD by 'LDL category' was graphically displayed using a Kaplan-Meier curve. Second, we report absolute disease risks (events per 1000 person-years) for each LDL category for the entire sample, and stratified by sex and by age group ( $<60$ and $\geq 60$ years). Third, Cox proportional hazard models were used to relate each LDL group to incident CVD, thereby adjusting for age, sex, systolic blood pressure, antihypertensive medication, smoking and diabetes mellitus. In secondary analyses, we additionally adjusted for presence versus absence of carotid ultrasound abnormality (defined as above) and for circulating CRP and BNP levels. In these models, we compared LDL categories 2-5 (each category considered separately) with LDL category 1 (usual LDL-C $<100 \mathrm{mg} / \mathrm{dL}$, no LLT). Fourth, we specifically compared treated with untreated individuals with similar LDL levels. Thus, we calculated multivariable-adjusted HR for incident CVD for group 5 (using group 4 as the referent) and for group 3 (using groups $1+2$ combined as the referent).

\section{RESULTS}

The baseline characteristics of the overall study sample and stratified by LDL group and LLT status are displayed in table 1. Overall, 302 participants (groups 3 and 5; $10 \%$ of the overall sample) were on lipid-lowering medications, and 170 treated individuals (group 3; 56\% of those on treatment) were controlled to a blood LDL level below $130 \mathrm{mg} / \mathrm{dL}$. Clinical, biochemical and ultrasound measures of the carotid arteries in the sample with available carotid ultrasound information $(n=2917)$ are provided in online supplementary table 1 , and the CVD baseline risk (10-year probability of a CVD event $)^{15}$ by LDL group is displayed in online supplementary figure 1. The 10-year probabilities for a CVD event in treated individuals (groups 3 and 5) were statistically significantly greater than in untreated individuals (groups 1, 2 and 4; $\mathrm{P}<0.0001$ ). More detailed information regarding individuals on LLT, including information on the average LDL-C level across exams 1-6 and the average duration of LLT, is provided in online supplementary table 2 . As expected, individuals in group 5 (LDL $\geq 130 \mathrm{mg} / \mathrm{dL}$ on treatment) had the highest LDL levels across exams 1-6 and across all exams before LLT was initiated $(175 \mathrm{mg} / \mathrm{dL}$; online supplementary table 2). In group 3, more than $80 \%$ of participants were on statins, whereas the proportion of statin users was smaller $(\sim 64 \%)$ in group 5.

\section{Association of LDL group with subclinical disease in the carotid arteries}

In age- and sex-adjusted and in multivariable-adjusted models, individuals on lipid-lowering medication (groups 3 and 5) and individuals with an untreated LDL above $130 \mathrm{mg} / \mathrm{dL}$ (group 4) had significantly higher ORs for carotid ultrasound abnormalities as compared with individuals in the referent group (usual $\mathrm{LDL}<100 \mathrm{mg} / \mathrm{dL}$; figure 1A,B). Furthermore, individuals on treatment who reach LDL levels $<130 \mathrm{mg} /$ dL (group 3) had statistically significantly increased odds for subclinical carotid ultrasound abnormalities as compared with untreated individuals with similar LDL levels (groups $1+2$ combined; OR: $1.95,95 \%$ CI 1.34 to $2.84, \mathrm{P}=0.0005)$. No statistically significant differences between treated individuals with LDL $\geq 130$ (group 5) and untreated individuals with comparable LDL levels (group 4; OR: 1.04, 95\% CI 0.69 to $1.57, \mathrm{P}=0.86$ ) could be observed.

\section{Residual risk for incident CVD in individuals on LLT}

During a median follow-up of 13.7 years $(Q 1=11.5$ years, Q3=14.9 years), 548 (255 women) out of 3012 participants developed an incident CVD event. The KaplanMeier curves for survival free of CVD by LDL groups are displayed in figure $2(\log -r a n k \mathrm{P}<0.0001)$. Table 2 displays the absolute disease risks by LDL group for the entire sample and stratified by sex and age group $(<60$ and $\geq 60$ years). Individuals on LLT (group 3 and group 5) had substantial residual cardiovascular risk for incident CVD that is, in the overall sample, approximately three times the absolute disease risk observed in group 1 (LDL below $100 \mathrm{mg} / \mathrm{dL}$, no LLT; table 2 'crude event rates'). In individuals below 60 years of age, the absolute disease risks in groups 3 and 5 (on LLT) were almost sixfold and fourfold, respectively, the risk in the referent group (table 2). In the age group $\geq 60$ years, the absolute cardiovascular risk rose for groups on LLT, but also the absolute risk in the referent group 1 increased, so that the relative risks in groups 2-5, compared with group 1, diminished with age. Furthermore, absolute CVD risks were slightly higher in men as compared with women for most LDL groups (table 2).

\section{Impact of adjusting for potential covariates, subclinical atherosclerosis of the carotid artery and biomarker levels on residual CVD risk}

Compared with reference group 1, LDL groups 3-5 displayed a statistically significantly increased relative hazard for incident CVD in multivariable-adjusted models (figure 3). Adjustment for subclinical atherosclerosis in the carotid arteries only modestly reduced 


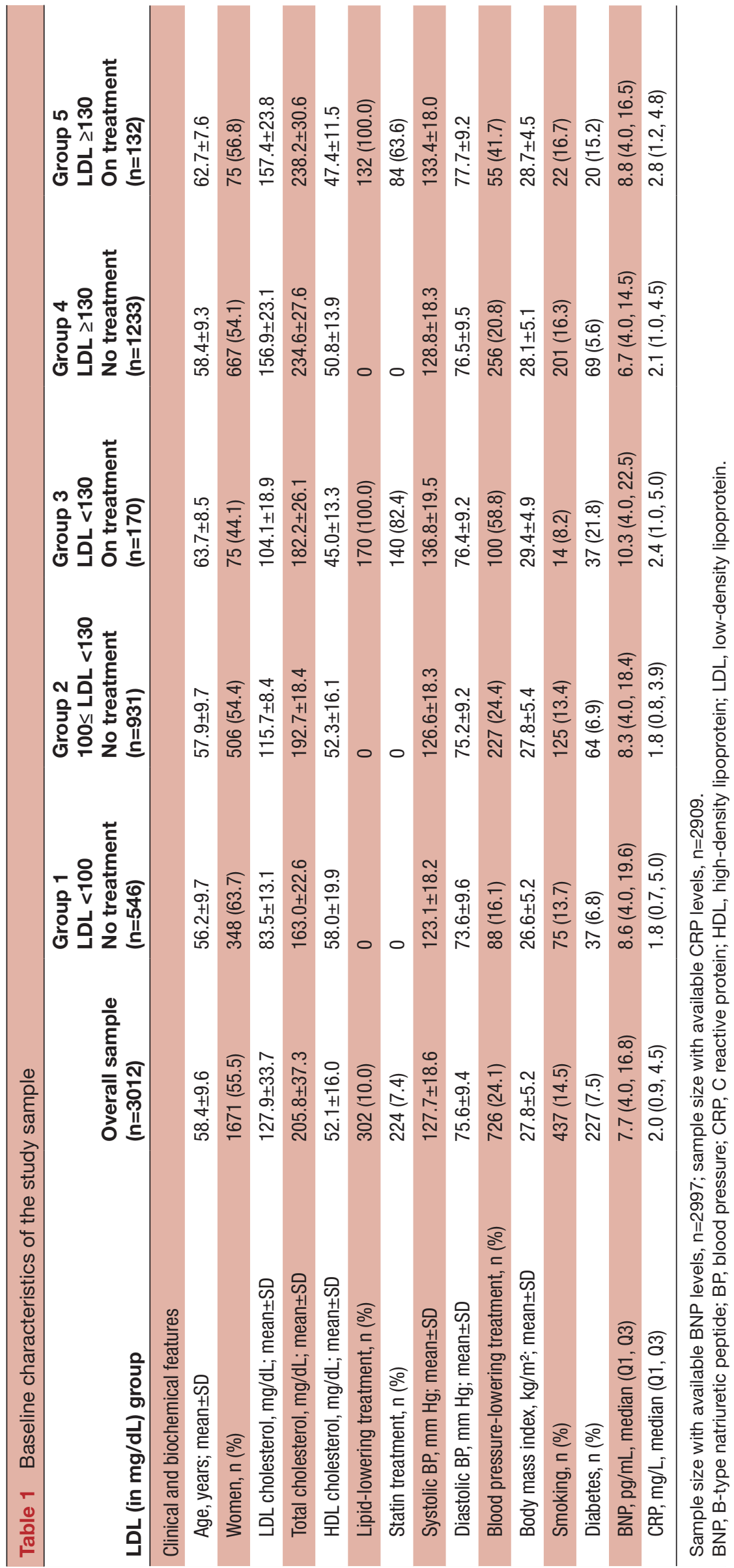


A

Odds ratio for carotid ultrasound abnormality (age- and sex-adjusted)

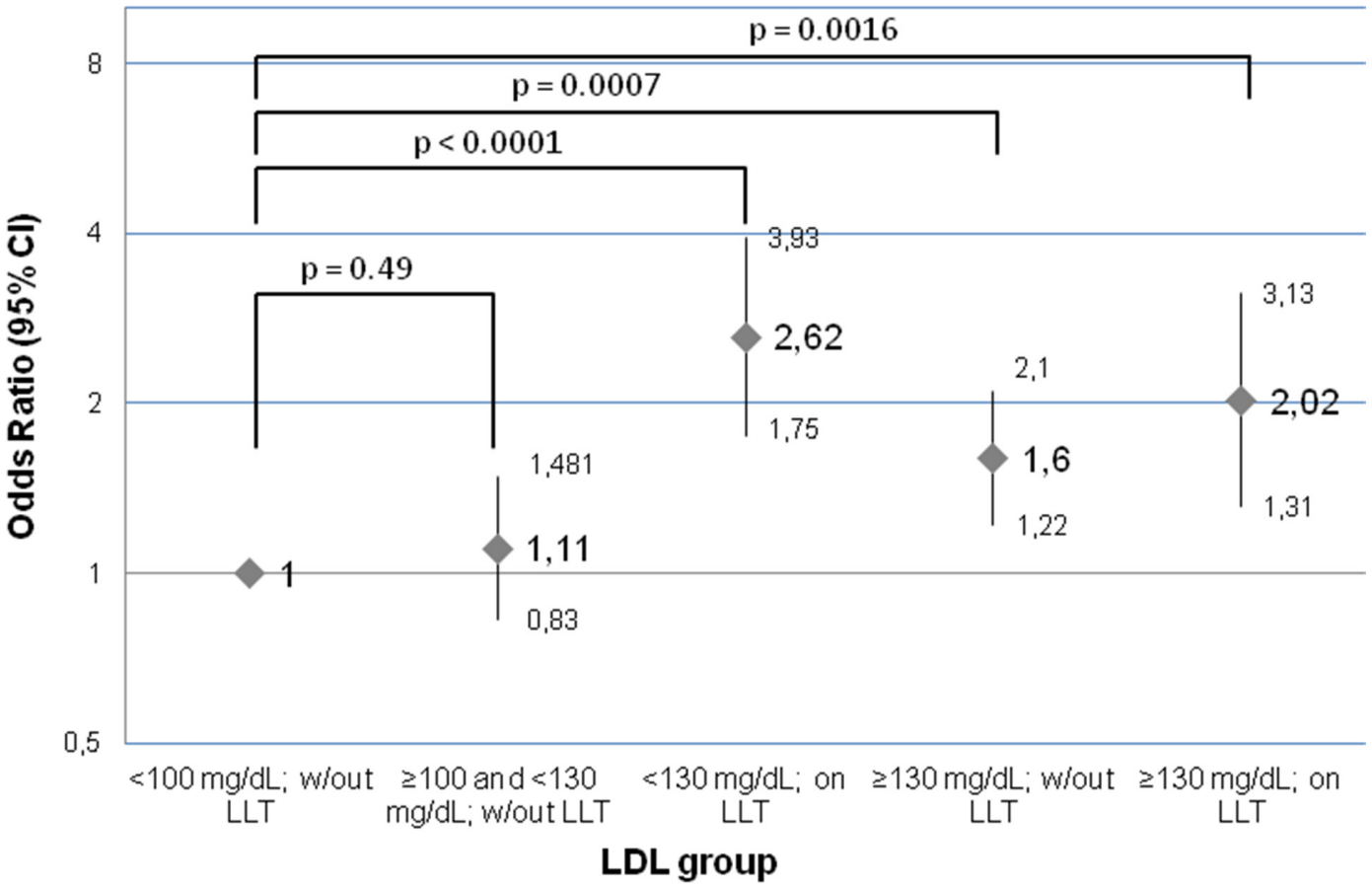

B

Odds ratio for carotid ultrasound abnormality (multivariable-adjusted)

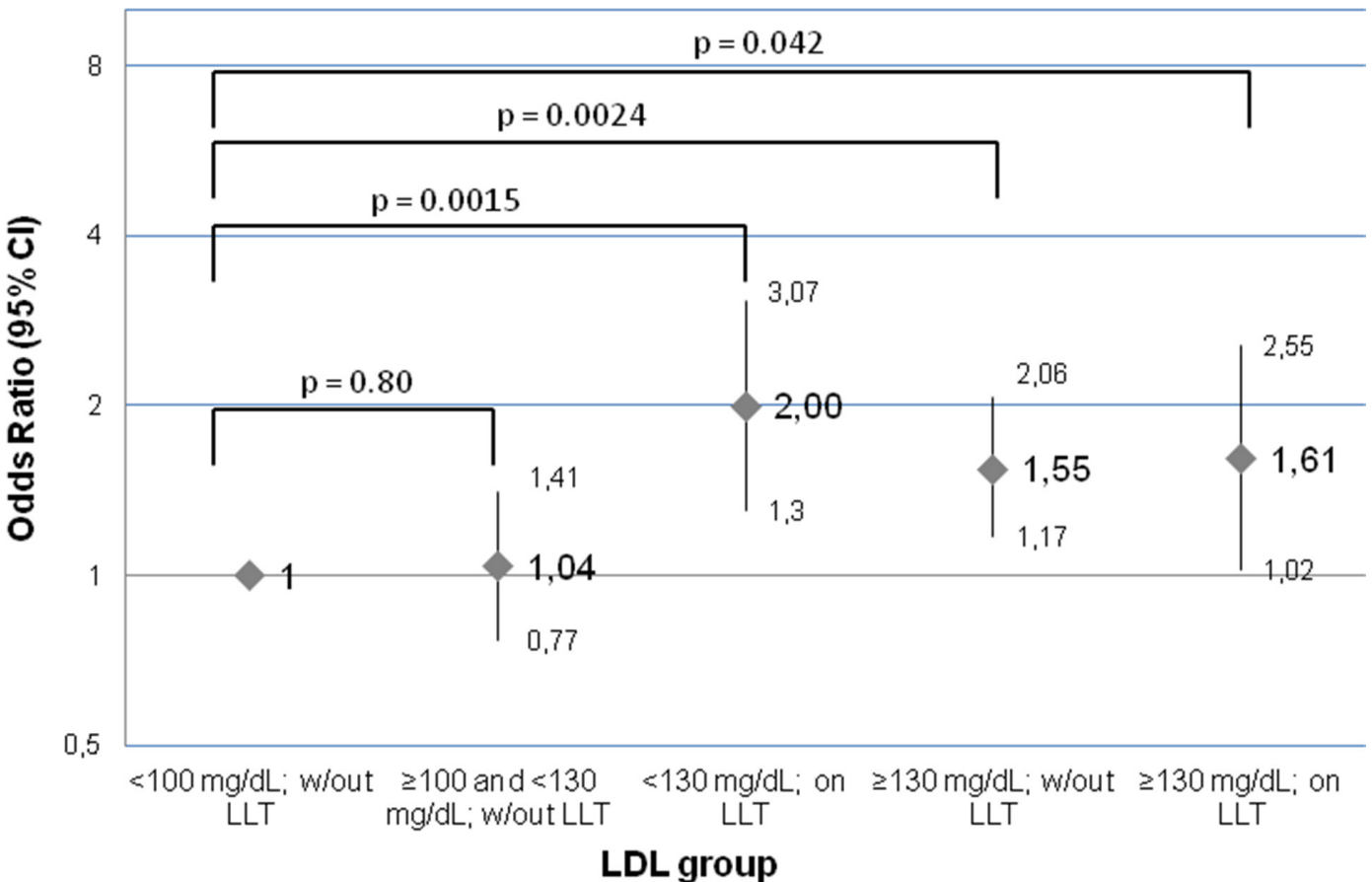

Figure 1 OR for the binary trait 'carotid ultrasound abnormality' in an age- and sex-adjusted model (A), as well as in a multivariable-adjusted model (B), including age, sex, systolic blood pressure, antihypertensive medication, smoking and diabetes mellitus. 'Carotid ultrasound abnormality' was defined as (1) increased ( $\geq 80^{\text {th }}$ sex-specific percentile) carotid IMT, a combined standardised measure including information from the internal and common carotid arteries, (2) an extreme increase of the common carotid IMT $\geq 1 \mathrm{~mm}$, or (3) significant stenosis ( $\geq 25 \%$ narrowing) of the common or internal carotid arteries, consistent with prior publications. ${ }^{1314}$ IMT, intima media thickness; LDL, low-density lipoprotein; LLT, lipid-lowering treatment; w/out, without. 


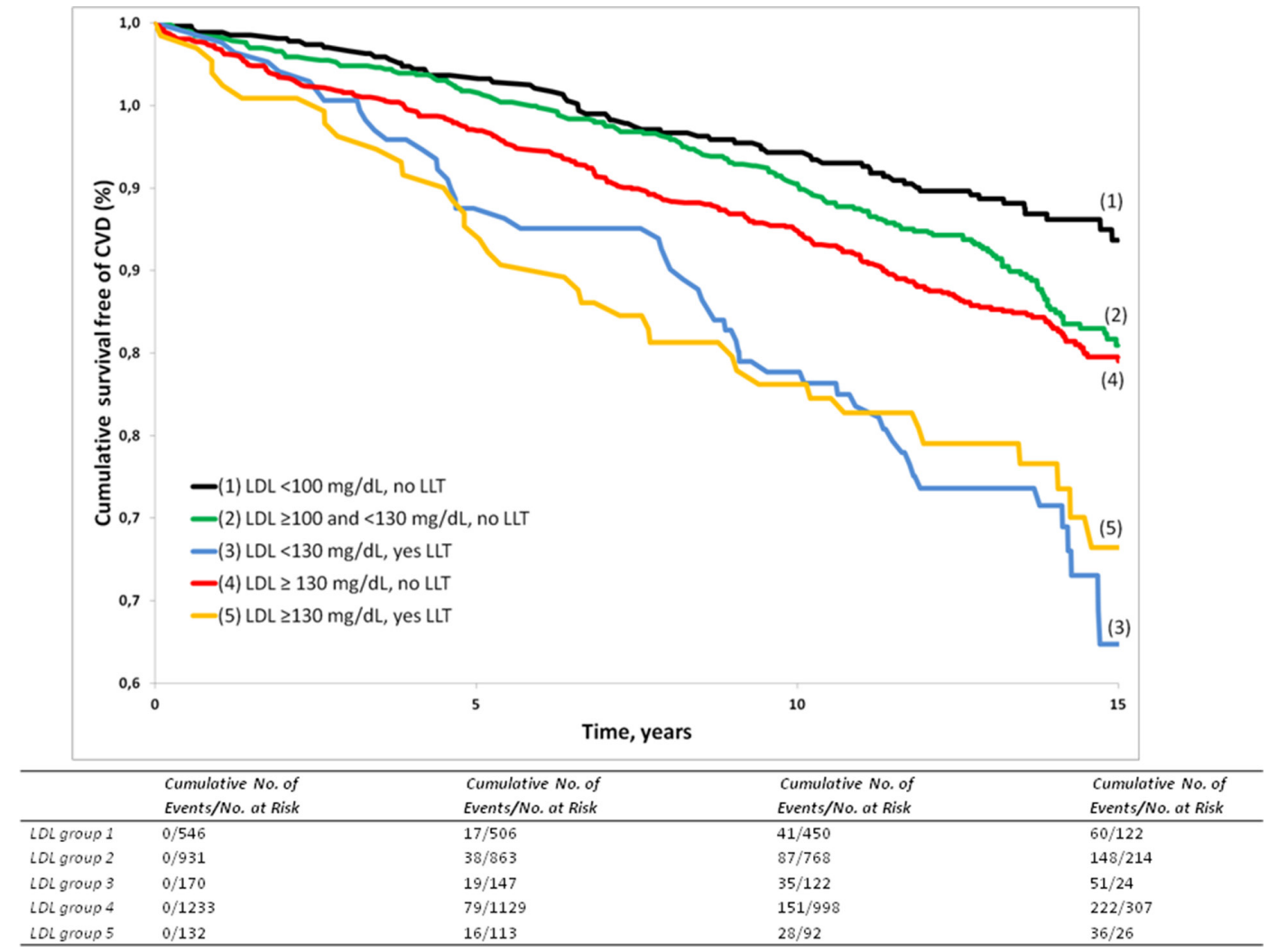

Figure 2 Survival free of CVD, stratified by LDL group. CVD, cardiovascular disease; LDL, low-density lipoprotein; LLT, lipidlowering treatment.

these HRs by up to $~ 5 \%$, and further adjustment for CRP and BNP had no relevant effect on the effect estimates (table 3).

Compared with untreated individuals with similar LDL (groups $1+2$ combined), individuals on treatment who reach $\mathrm{LDL}<130 \mathrm{mg} / \mathrm{dL}$ (group 3) had statistically increased hazards for incident CVD in an age- and sex-adjusted model (HR: 1.44, 95\% CI 1.06 to $1.96, \mathrm{P}=0.02$ ), but this difference was no longer statistically significant on multivariable adjustment (HR: $1.20,95 \%$ CI 0.88 to 1.64 , $\mathrm{P}=0.25$ ). With respect to individuals with $\mathrm{LDL} \geq 130 \mathrm{mg}$ / $\mathrm{dL}$, treated individuals (group 5) were not different from untreated individuals (group 4) with respect to new-onset CVD in age- and sex-adjusted (HR: 1.31, 95\% CI 0.92 to 1.87, $\mathrm{P}=0.13$ ) and in multivariable-adjusted (HR: 1.09, $95 \%$ CI 0.76 to $1.55, \mathrm{P}=0.65$ ) models.

\section{DISCUSSION}

In a large community-based sample, we provide absolute CVD risk estimates for the entire sample and stratified by age and by sex for different categories of LDL levels, representing different treatment intensities of LLT. Furthermore, we assessed potential mechanisms by which the residual risk could be mediated: subclinical carotid disease, neurohormonal activation or chronic inflammation. In addition, we describe the subclinical disease burden at the carotid arteries associated with different lipid levels in the community, stratified by treatment status.

\section{Principal observations}

First, subclinical disease burden and risk for incident CVD rose with increasing LDL category. Second, individuals who reach LDL levels $<130 \mathrm{mg} / \mathrm{dL}$ on treatment (group 3) as well as individuals with LDL $\geq 130 \mathrm{mg} / \mathrm{dL}$ (groups 4 and 5) displayed greater carotid atherosclerotic disease burden, as compared with untreated individuals with usual LDL $<100 \mathrm{mg}$ / $\mathrm{dL}$ (group 1). Third, individuals on lipid-lowering medication (groups 5 and 3) had a substantial residual absolute risk for incident CVD that was about three times the risk observed in individuals with LDL $<100 \mathrm{mg} / \mathrm{dL}$ who were not on treatment. Residual CVD risk rose substantially with age, and men had slightly higher absolute CVD risk than women in most LDL categories. Fourth, part of this increased risk was due to worse cardiovascular risk profiles in groups 3-5 (table 1). However, on adjustment for traditional CVD risk factors, individuals on LLT who reach LDL $<130 \mathrm{mg} / \mathrm{dL}$ still had about $50 \%$ greater hazards for CVD as compared with the referent group. Similarly, groups 4 and 5 displayed statistically significant HRs for CVD (compared with group 1), taking potential confounders into account. Fifth, adjustment for subclinical carotid disease burden only moderately attenuated these HRs (by about 5\%) consistent with partial mediation of the residual CVD risk by the burden of subclinical atherosclerosis. Further 


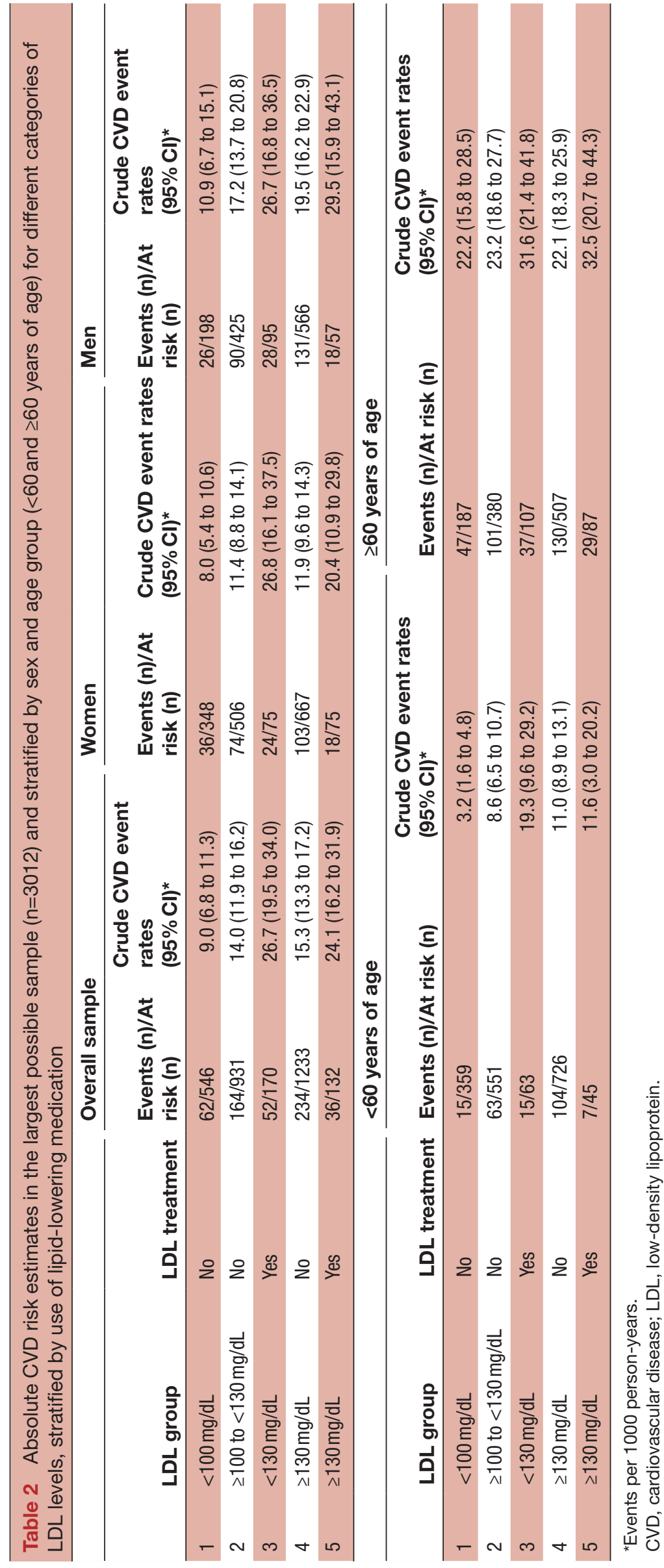




\section{Hazard ratios of LDL groups for incident CVD (multivariable-adjusted)}

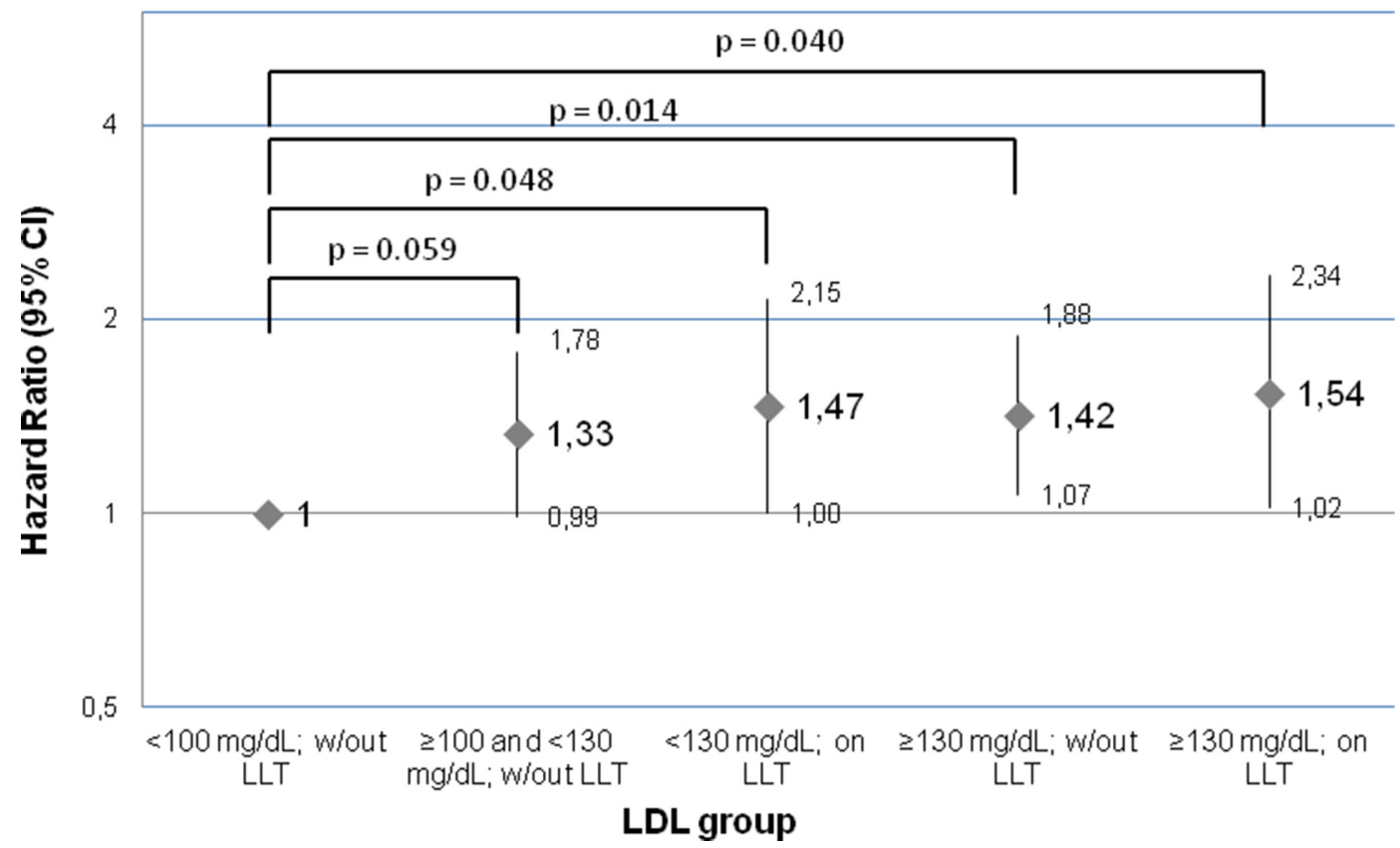

Figure 3 HRs for incident CVD by LDL group, adjusted for age, sex, systolic blood pressure, antihypertensive medication, smoking and diabetes mellitus. CVD, cardiovascular disease; LDL, low-density lipoprotein; LLT, lipid-lowering treatment; w/out, without.

adjustment for CRP and BNP did not significantly alter the risk estimates.

\section{In the context of the published literature}

The association between lipid measures and CVD risk is well established in the epidemiological and clinical literature. ${ }^{17}$ Numerous clinical trials have demonstrated that lowering of LDL-C through pharmacological or lifestyle interventions reduces the risk for recurrent CVD and mortality in patients with established $\mathrm{CVD}^{118}$ or in the primary prevention setting. ${ }^{2}{ }^{20}$ Furthermore, clinical trials have shown that statins-by lowering LDL-also improve subclinical CVD measures. A recent meta-analysis of 11 clinical trials reported beneficial effects of statins on carotid atherosclerosis. ${ }^{21}$

Despite clinically and statistically significant reductions in clinical and subclinical CVD risk in the LLT arm compared with the respective comparison groups, many clinical trials reported a relevant residual cardiovascular risk in individuals receiving LLT, with the magnitude of the residual risk depending on the exact patient sample, the duration of follow-up, the type (generation) and dosage of treatment used, the target LDL levels that have been reached, and the exact endpoint investigated in the study. ${ }^{1} 181922-25$ Clinical trials have relatively strict inclusion and exclusion criteria so that it is unclear whether the observations from clinical trials regarding the residual cardiovascular risk on LLT are applicable to the general population. ${ }^{26}$

In our unselected sample from the community, we confirm a substantial residual absolute CVD risk in individuals on LLT (group 3 and group 5). Individuals in these groups had about three times the risk of the referent group 1 (untreated LDL $<100 \mathrm{mg} / \mathrm{dL}$ ) in the overall sample. In individuals below 60 years of age, the ratios of the absolute disease risks in those on LLT and those in the referent group 1 were even more pronounced.

Furthermore, we explored the subclinical disease burden at the carotid arteries associated with various LDL levels, stratified by LLT status. Individuals on LLT and with greater LDL levels displayed statistically significantly greater odds for carotid atherosclerosis on ultrasound, including increased IMT and stenoses, ${ }^{13}$ consistent with other observational studies. ${ }^{27} 28$ In addition, we evaluated to what extent the increased hazards for CVD in LDL groups 3-5 (figure 3) were attributable to subclinical atherosclerosis at the carotid arteries and to systemic inflammation and neurohormonal activation. Our observations indicate that adjustment for subclinical carotid atherosclerosis only modestly attenuated the relative risk estimates, consistent with the concept that a modest proportion of the increased risk is attributable to subclinical atherosclerosis. Additional adjustment for established biomarkers of systemic inflammation (CRP) and neurohormonal activation (BNP) had no significant effect on the risk estimates.

Important drivers for the elevated subclinical and clinical CVD risk in individuals on LLT with LDL levels $<130 \mathrm{mg} / \mathrm{dL}$ (group 3) are unfavourable levels for other risk factors, including systolic blood pressure, body mass index, diabetes and HDL in participants in 
this group (table 1). The greater CVD risk at baseline in individuals on LLT (also reflected in online supplementary figure 1) may reflect indication bias (confounding by indication; higher risk individuals are more likely to be treated by their physicians), as expected in observational (non-randomised) studies assessing residual risk on treatments. ${ }^{29}$ Indeed, one of the factors contributing to the decision whether LLT is initiated in a given patient is the overall CVD risk over a 10-year time horizon, which takes levels of several risk factors into account. ${ }^{17}$ On the other hand, and as a clinical consequence, these observations underscore the importance of monitoring all traditional risk factors in individuals on LLT, even if people reach LDL levels below $130 \mathrm{mg} / \mathrm{dL}$.

It also has to be kept in mind that LDL-cholesterol, despite being an established lipid measure and CVD risk factors, might mirror LDL-related residual CVD risk not ideally, particularly in subgroups of the population, where other lipid measures (such as apolipoprotein B (ApoB) concentrations) provide discordant information. In the Women's Health Study, approximately one in five women were discordant for LDL-C and ApoB; and CVD risk in these individuals was underestimated or overestimated, when estimations were solely based on LDL-C. ${ }^{30}$ Also in the Framingham Offspring cohort, ApoB levels provided additional information about CVD risk, beyond established lipid measures, in individuals, where the observed ApoB levels were discordant to the expected ApoB concentrations, as predicted based on circulating LDL-C. ${ }^{31}$ Furthermore, in Jupiter trial participants (individuals with $\mathrm{LDL}<130 \mathrm{mg} / \mathrm{dL}$ and high-sensitivity C-reactive protein (hsCRP) $\geq 2.0 \mathrm{mg} / \mathrm{L}$ ), ApoB levels, but not LDL-C, were associated with incident CVD in the placebo arm. $^{32}$ In the on-statin arm, however, ApoB failed to reach significance (HR: $1.20,95 \%$ CI 0.98 to 1.46 for incident CVD) ${ }^{32}$

\section{Strengths and limitations}

The strengths of our investigation include the large community-based sample, the careful and comprehensive characterisation of our study participants, including measures of subclinical atherosclerosis at the carotid arteries and of biomarkers of systemic inflammation and neurohormonal activation, and the prospective follow-up for incident CVD (median follow-up, 13.7 years). The following limitations merit consideration. We assigned participants to LDL groups based on a single LDL measurement and a single assessment of the intake of lipid-lowering medication. This might result in some misclassification, which is likely to be non-differential; this would bias us towards the null hypothesis of no difference between the groups in risk of outcome events. In our analyses we did not differentiate between different types of lipid-lowering medication, dosages and durations of treatment. Unfortunately, information on the generation of statin taken or on statin dose was not available in our sample. Furthermore, the prescription of LLT is determined by circulating lipid concentrations and by 
the overall burden of CVD risk factors. Thus, individuals on LLT in the community might a priori be at higher CVD risk as compared with individuals not receiving such medication (confounding by indication) ${ }^{29}$ Finally, we have a relatively small number of individuals on statins. However, these individuals were well phenotyped and had a relatively long follow-up period well beyond that of typical clinical trials of LLT.

In conclusion, we observed a substantial residual cardiovascular risk in individuals on lipid-lowering medication in the community, partly explained by an adverse profile of other CVD risk factors and in part (by about $5 \%$ ) by carotid subclinical atherosclerosis in these people. These observations emphasise the necessity to closely monitor all standard CVD risk factors in individuals on LLT, even when LDL levels are lowered effectively.

Contributors WL and RSV drafted the article. DME and MGL performed the statistical analyses. All authors interpreted the data and reviewed and edited the manuscript for important intellectual content.

Funding This work was supported by the Framingham core contract N01-HC25195 and HHSN268201500001I (to RSV).

\section{Competing interests None declared.}

Ethics approval The study protocol was approved by the Institutional Review Committee of Boston University Medical Center.

Provenance and peer review Not commissioned; externally peer reviewed.

Data sharing statement The data from the Framingham Heart Study used for this project are available in the NHLBI data repository BioLINCC ().

Open Access This is an Open Access article distributed in accordance with the Creative Commons Attribution Non Commercial (CC BY-NC 4.0) license, which permits others to distribute, remix, adapt, build upon this work non-commercially, and license their derivative works on different terms, provided the original work is properly cited and the use is non-commercial. See: http://creativecommons.org/ licenses/by-nc/4.0/

(c) Article author(s) (or their employer(s) unless otherwise stated in the text of the article) 2018. All rights reserved. No commercial use is permitted unless otherwise expressly granted.

\section{REFERENCES}

1. Group SSSS. Randomised trial of cholesterol lowering in 4444 patients with coronary heart disease: the Scandinavian Simvastatin Survival Study (4S). Lancet 1994;344:1383-9.

2. Colhoun HM, Betteridge DJ, Durrington PN, et al. Primary prevention of cardiovascular disease with atorvastatin in type 2 diabetes in the Collaborative Atorvastatin Diabetes Study (CARDS): multicentre randomised placebo-controlled trial. Lancet 2004;364:685-96.

3. Mihaylova B, Emberson J, Blackwell L, et al. The effects of lowering LDL cholesterol with statin therapy in people at low risk of vascular disease: meta-analysis of individual data from 27 randomised trials. Lancet 2012;380:581-90.

4. Taylor F, Huffman MD, Macedo AF, et al. Statins for the primary prevention of cardiovascular disease. Cochrane Database Syst Rev 2013;1:CD004816.

5. Parish E, Bloom T, Godlee F. Statins for people at low risk. BMJ 2015;351:h3908.

6. Krumholz HM. Statins evidence: when answers also raise questions. BMJ 2016;354:i4963.

7. Horton R. Offline: Lessons from the controversy over statins. Lancet 2016;388:1040.

8. Kannel WB, Feinleib M, McNamara PM, et al. An investigation of coronary heart disease in families. The Framingham offspring study. Am J Epidemiol 1979;110:281-90.

9. Andersson C, Lyass A, Vasan RS, et al. Long-term risk of cardiovascular events across a spectrum of adverse major plasma lipid combinations in the Framingham Heart Study. Am Heart $J$ 2014;168:878-83.
10. Maas R, Xanthakis V, Polak JF, et al. Association of the endogenous nitric oxide synthase inhibitor ADMA with carotid artery intimal media thickness in the Framingham Heart Study offspring cohort. Stroke 2009:40:2715-9.

11. Polak JF, Pencina MJ, Pencina KM, et al. Carotid-wall intimamedia thickness and cardiovascular events. N Engl J Med 2011;365:213-21.

12. Fox CS, Cupples LA, Chazaro I, et al. Genomewide linkage analysis for internal carotid artery intimal medial thickness: evidence for linkage to chromosome 12. Am J Hum Genet 2004;74:253-61.

13. Lieb W, Enserro DM, Sullivan LM, et al. Residual Cardiovascular Risk in Individuals on Blood Pressure-Lowering Treatment. J Am Heart Assoc 2015;4:e002155.

14. Ingelsson E, Sullivan LM, Murabito JM, et al. Prevalence and prognostic impact of subclinical cardiovascular disease in individuals with the metabolic syndrome and diabetes. Diabetes 2007;56:1718-26.

15. D'Agostino RB, Vasan RS, Pencina MJ, et al. General cardiovascular risk profile for use in primary care: the Framingham Heart Study. Circulation 2008:117:743-53.

16. Yang C, Sun Z, Li Y, et al. The correlation between serum lipid profile with carotid intima-media thickness and plaque. BMC Cardiovasc Disord 2014:14:181.

17. Stone NJ, Robinson JG, Lichtenstein AH, et al. 2013 ACC/ AHA guideline on the treatment of blood cholesterol to reduce atherosclerotic cardiovascular risk in adults: a report of the American College of Cardiology/American Heart Association Task Force on Practice Guidelines. Circulation 2014:129:S1-45.

18. Sacks FM, Pfeffer MA, Moye LA, et al. The effect of pravastatin on coronary events after myocardial infarction in patients with average cholesterol levels. Cholesterol and Recurrent Events Trial investigators. N Engl J Med 1996;335:1001-9.

19. Downs JR, Clearfield M, Weis S, et al. Primary prevention of acute coronary events with lovastatin in men and women with average cholesterol levels: results of AFCAPS/TexCAPS. Air Force/Texas Coronary Atherosclerosis Prevention Study. JAMA 1998;279:1615-22.

20. Collins R, Reith C, Emberson J, et al. Interpretation of the evidence for the efficacy and safety of statin therapy. Lancet 2016;388:2532-61.

21. Bedi US, Singh M, Singh PP, et al. Effects of statins on progression of carotid atherosclerosis as measured by carotid intimal-medial thickness: a meta-analysis of randomized controlled trials. J Cardiovasc Pharmacol Ther 2010;15:268-73.

22. Ridker PM, Danielson E, Fonseca FA, et al. Rosuvastatin to prevent vascular events in men and women with elevated C-reactive protein. N Engl J Med 2008;359:2195-207.

23. Shepherd J, Cobbe SM, Ford I, et al. Prevention of coronary heart disease with pravastatin in men with hypercholesterolemia. West of Scotland Coronary Prevention Study Group. N Engl J Med 1995;333:1301-7.

24. Yusuf S, Bosch J, Dagenais G, et al. Cholesterol Lowering in Intermediate-Risk Persons without Cardiovascular Disease. N Engl J Med 2016;374:2021-31.

25. Collins R, Armitage J, Parish S, et al. Effects of cholesterol-lowering with simvastatin on stroke and other major vascular events in 20536 people with cerebrovascular disease or other high-risk conditions. Lancet 2004;363:757-67.

26. Hannan EL. Randomized clinical trials and observational studies: guidelines for assessing respective strengths and limitations. JACC Cardiovasc Interv 2008;1:211-7.

27. Salonen R, Salonen JT. Determinants of carotid intima-media thickness: a population-based ultrasonography study in eastern Finnish men. J Intern Med 1991;229:225-31.

28. Tattersall MC, Gassett A, Korcarz CE, et al. Predictors of carotid thickness and plaque progression during a decade: the Multi-Ethnic Study of Atherosclerosis. Stroke 2014;45:3257-62.

29. Signorello LB, McLaughlin JK, Lipworth L, et al. Confounding by indication in epidemiologic studies of commonly used analgesics. Am J Ther 2002;9:199-205.

30. Mora S, Buring JE, Ridker PM. Discordance of low-density lipoprotein (LDL) cholesterol with alternative LDL-related measures and future coronary events. Circulation 2014;129:553-61.

31. Pencina MJ, D'Agostino RB, Zdrojewski T, et al. Apolipoprotein B improves risk assessment of future coronary heart disease in the Framingham Heart Study beyond LDL-C and non-HDL-C. Eur J Prev Cardiol 2015;22:1321-7.

32. Lawler PR, Akinkuolie AO, Chu AY, et al. Atherogenic lipoprotein determinants of cardiovascular disease and residual risk among individuals with low low-density lipoprotein cholesterol. J Am Heart Assoc 2017;6:e005549. 